\title{
Impacto de la Metodología de Aprendizaje-Servicio del Programa de Grado de Capacitación de Profesores de Inglés Extranjero para estudiantes chilenos desfavorecidos
}

\author{
Impact of Community Service Learning Methodology on Underprivileged chilean \\ Undergraduate English Foreign Language Teacher Training Program Students
Impacto da Metodologia de Aprendizagem em Serviço do Programa de Graduação para
Professores de Inglês Estrangeiros para estudantes chilenos desfavorecidos

\author{
Tamara Cortés Seitz ${ }^{a}$ \\ ${ }^{a}$ Universidad Católica Silva Henríquez. Chile. \\ tcortess@ucsh.cl
}

\section{RESUMEN}

Si bien investigaciones han sido realizadas en los efectos que el Aprendizaje y Servicio tiene en las áreas de enfermería, medicina e ingeniería, existe muy poca información de los efectos que esta metodología tiene en estudiantes de pedagogía en inglés. El presente estudio, el cual fue realizado en un periodo de 4 años, tiene como objetivo identificar el impacto que esta metodología tiene en estudiantes chilenos de pedagogía en inglés en situación de alto riesgo social. Los participantes fueron 90 estudiantes de segundo y tercer año de la carrera de pedagogía en inglés y 200 socios comunitarios. Las necesidades de la comunidad al igual que su nivel de inglés fueron identificados con una prueba de análisis de necesidades y una prueba de diagnóstico respectivamente. Los estudiantes de pregrado completaron diarios de reflexiones en donde contestaban cuatro preguntas para identificar los efectos que esta metodología tuvo en ellos, mientras que los socios comunitarios completaron una encuesta de satisfacción. Ambos grupos también participaron de sesiones de conversación para indagar aún más en las respuestas entregadas. El análisis de los datos recopilados muestra que esta metodología tuvo un impacto positivo en los estudiantes cuando se hace referencia a la profesionalización de su carrera y la aplicación de la teoría a la realidad. Los resultados de la comunidad si bien fueron positivos, están incompletos pues no se pudo realizar las sesiones de conversación.

Palabras claves: Formación docente, Aprendizaje y servicio, Enseñanza de una lengua.

\begin{abstract}
Although research has been conducted on the effects of Community Service learning in nursing, medical and engineering students, there is very little information about the effects that this methodology has on English as a Foreign Language (EFL) teacher training students. The present study, which was conducted over four years, attempted to identify the impact that this methodology had on underprivileged Chilean EFL teacher training undergraduate students. The participants were 90 sophomore and junior EFL teacher training students and 200 community members. The needs of the community as well as the English level were identified with a needs analysis and placement test. Undergraduates were asked to answer four questions in the form of reflection logs to identify the effects that this methodology had on them while a satisfaction survey was used to identify the impact the methodology had on the community members. The analysis of the answers in the logs revealed that undergraduate students became aware of the relevance of identifying the community's needs, having clear objectives, preparing material and activities before designing a course and teaching a class. This methodology
\end{abstract}


showed to have a positive effect on the teacher training students when referring to their professionalization and application of theory and concepts to reality. For the community, the results were positive but further research is needed to identify the full impact in the community members.

Key words: Teacher education, Study service, Language instruction.

\section{RESUMO}

Embora pesquisas tenham sido realizadas sobre os efeitos que Service Learning tem nas áreas de enfermagem, medicina e engenharia, há muito pouca informação sobre os efeitos que esta metodologia tem em alunos de pedagogia em inglês. O presente estudo, realizado ao longo de 4 anos, visa identificar o impacto que esta metodologia tem sobre os alunos chilenos de pedagogia da língua inglesa em situação de alto risco social. Os participantes foram 90 alunos do segundo e terceiro ano de pedagogia de inglês e 200 parceiros da comunidade. As necessidades da comunidade, bem como seu nível de inglês, foram identificadas com um teste de análise de necessidades e um teste de diagnóstico, respectivamente. Os alunos de graduação preencheram diários de reflexão nos quais responderam a quatro perguntas para identificar os efeitos que essa metodologia teve sobre eles, enquanto os parceiros da comunidade responderam a uma pesquisa de satisfação. Ambos os grupos também participaram de sessões de bate-papo para investigar melhor as respostas fornecidas. A análise dos dados coletados mostra que essa metodologia teve um impacto positivo nos alunos no que se refere à profissionalização de sua carreira e à aplicação da teoria à realidade. Embora os resultados da comunidade tenham sido positivos, eles estão incompletos porque as sessões de conversação não puderam ser realizadas.

Palavras-chave: Formação de professores, Aprendizagem e serviço, Ensino de uma língu.

\section{INTRODUCCIÓN}

Como profesora que trabaja en una institución de educación superior que prepara a futuros docentes que dirigirán y darán forma a las generaciones más jóvenes, entiendo que la enseñanza de hoy se ha convertido en un gran desafío, no solamente porque tenemos que asegurarnos de que los estudiantes han dominado las teorías y contenidos de sus clases, sino también que han desarrollado las habilidades emocionales y cívicas que les permitirán ser socialmente más responsables. El reto que hoy en día tenemos los profesores y las universidades es formar profesionales que no sólo cuenten con altos estándares académicos, sino que también posean un propósito social profundo y claro.

Hoy en día la sociedad requiere de más profesionales que puedan entender y aplicar contenidos y teorías utilizando herramientas que les permitan resolver problemas en escenarios complejos. Esto significa que las universidades, como instituciones académicas y de formación profesional, deben contar con profesores expertos que puedan capacitarse y ayudar a desarrollar las competencias de los estudiantes, permitiéndoles utilizar estas competencias o habilidades de manera responsable y teniendo en cuenta la realidad social actual. Por lo tanto, la formación de profesionales debe incluir casos en los que los estudiantes puedan mejorar las diferentes habilidades al desarrollar conocimientos de la manera más significativa, mientras aprenden a tomar decisiones efectivas en las diferentes áreas de sus campos profesionales. Es importante recordar que las Universidades tienen la responsabilidad y la obligación de ayudar a formar a los ciudadanos que realizan sus prácticas profesionales al servicio de la sociedad, por lo que es necesario encontrar metodologías innovadoras y estrategias de enseñanza que ayuden con esta importante tarea. De hecho, en el 2015 la UNESCO declaró que era responsabilidad de las universidades no sólo fomentar la responsabilidad social sobre sus estudiantes, sino también "promover el aprendizaje 
permanente mediante el establecimiento de asociaciones conjuntas de investigación y formación universitarias/comunitarias y llevar los servicios de las universidades a grupos externos" (p. 7). En este escenario, la metodología de Aprendizaje-Servicio (AS) se presenta como una herramienta que permite a las instituciones de educación superior responder a las necesidades sociales y académicas, ofreciendo a los estudiantes la oportunidad de aprender haciendo. Esta idea, que es fundamental para la teoría de la educación de John Dewey (citado en Saltmarsh, 1996), considera el proceso de aprendizaje como una "práctica conducida deliberadamente" (p. 14), donde hay una interacción activa y deliberada entre "acción y hacer, por un lado, y conocimiento y comprensión por el otro" (p. 15). En Chile, mientras que la mayoría de las universidades chilenas cuentan con programas de divulgación comunitaria y/o trabajo de caridad, estas actividades no están relacionadas con el plan de estudios de los estudiantes, y muchos estudiantes se gradúan sin experimentar nunca el aprendizaje de servicio comunitario.

Antes de indagar acerca de la relevancia y los efectos que esta metodología ha tenido en la formación profesional a nivel universitario, primero tenemos que definir qué es la metodología del aprendizaje-servicio. En el informe publicado en 1993, la Alianza para el Aprendizaje de Servicios en la Reforma Educativa presentó una definición del aprendizajeservicio y lo considera un método de enseñanza donde los jóvenes aprenden y desarrollan habilidades participando activamente en experiencias de servicio escolar y que responden a las necesidades reales de la comunidad. Estas actividades deben integrarse al currículo académico o escolar y ofrecer al alumno la oportunidad de utilizar las habilidades o poner en práctica los contenidos presentados en sus cursos en situaciones de la vida real. Bringle y Hatcher (1995 p. 112) definieron el aprendizaje-servicio como "una actividad de servicio organizada que satisface las necesidades de la comunidad y reflexiona sobre la actividad del servicio, de tal manera que se entienda mejor el contenido del curso, se tenga una apreciación más amplia de la disciplina y se obtenga mayor sentido de responsabilidad cívica". Wade (2000) amplía esta idea indicando que el aprendizaje-servicio es una metodología de enseñanza que ayuda a crear puentes entre los estudiantes y las instituciones que los educan, al combinar la enseñanza académica con prácticas o experiencias significativas de servicio comunitario a través de la conexión del plan de estudios y la comunidad. Puig (citado en Santos, Sotelino y Meledo, 2015) define esta metodología como una:

Propuesta educativa que combina procesos de aprendizaje y servicio a la comunidad en un proyecto que ha sido articulado de tal forma que los participantes están siendo formados mientras trabajan en las necesidades reales de la comunidad con el fin de mejorarla (p. 4).

Dos años más tarde, el autor redefine este concepto añadiendo que esta metodología "combina contenidos, habilidades y valores en una sola actividad que presta un servicio a la comunidad" (Puig, citado en Santos, Sotelino y Meledo, 2015, p. 4). En 2014, María Nieves Tapia, directora de CLAYSS, define a esta metodología como una propuesta pedagógica que permite a niños, adolescentes y adultos jóvenes desarrollar conocimientos y habilidades a través de actividades comunitarias (p. 17), e indica que el AS no se refiere a caridad o trabajo voluntario. La interacción con la comunidad fuera del aula de clase proporciona una excelente oportunidad para aprender y probar lo que funciona y lo que no funciona. A través de estas reflexiones, los futuros profesionales, así como sus formadores 
o profesores, deben reaccionar y evaluar si lo que se está enseñando es relevante, apropiado o útil para la sociedad actual (Santander, 2019, introducción).

Al utilizar esta metodología, el papel del estudiante cambia de pasivo a activo. En esta metodología los estudiantes son los protagonistas y lideran el proceso "no sólo participando en las comunidades", sino también "transformándose como ciudadanos comprometidos y activos" (Mitchel, 2008, p. 52). En la ceremonia de apertura del Seminario CLAYSS 2016, Maria Nieves Tapia destacó la importancia del papel activo que los alumnos deben desempeñar en esta metodología ya que son el eje principal, y continuó recordando a la audiencia que para que una actividad sea considerada como AS debe ser conducida fundamentalmente por los estudiantes y debe responder y satisfacer una necesidad real de la comunidad (Tapia, 2016). En su discurso de clausura, Tapia reiteró que el aprendizajeservicio proporciona un mayor y mejor aprendizaje para los estudiantes, ya que crea un contacto directo con la realidad a través de la identificación y resolución de problemas de la comunidad, creando así una instancia única para desarrollar los valores en los estudiantes. En otras palabras, aprendizaje-servicio es una metodología que ofrece un servicio comunitario desarrollado por estudiantes y destinado a cubrir las necesidades reales de la comunidad. Este servicio ha sido planificado e integrado institucionalmente en el plan de estudios para ofrecer a los estudiantes más oportunidades de práctica real mientras fortalecen el proceso de aprendizaje. Kahne, Westheimer y Rogers creen firmemente que:

Los estudiantes que realizan experiencias de servicio más extensas, así como aquellos cuyas experiencias están bien integradas en el plan de estudios, tienden a abordar los problemas sociales de una manera más compleja y exhaustiva que los no participantes o estudiantes (2000, p. 44).

Al referirse al uso de esta metodología en la educación superior, Vázquez (2015) afirma en la introducción de su artículo que la metodología de aprendizaje-servicio a nivel universitario es una herramienta que ayuda a desarrollar habilidades que resuelven el problema de sostenibilidad que enfrentan las instituciones de educación superior. Esto significa que el aprendizaje-servicio es una actividad de los estudiantes y que está adscrita a una actividad curricular que ofrece un servicio concreto, permanente y eficiente a un problema identificado por los estudiantes en una comunidad en particular, generando una relación bidireccional entre la comunidad y los estudiantes o centros educativos. Esta relación permite a las instituciones obtener una retroalimentación sobre la relevancia de sus programas y la eficacia de sus métodos de enseñanza, al tiempo que proporciona a los estudiantes oportunidades para implementar lo que han aprendido y reflexionar sobre su propia práctica profesional. De esta manera, los miembros de las instituciones educativas y las diversas comunidades trabajan juntos para lograr resultados que sean mutuamente beneficiosos. Esta modalidad combina el currículo académico y el servicio comunitario, ofreciendo a los participantes la oportunidad de estar acompañados y guiados por profesionales, expertos en sus materias, mientras realizan el trabajo comunitario. Como explica Santander, cuando se trata del papel de los profesores que utilizan esta metodología en su curso "los profesores guían, acompañan y motivan a los alumnos en este proceso, donde descubren y toman conciencia de cómo utilizar sus habilidades disciplinarias para el bien de una comunidad que requiere un servicio" (2019, p. 16).

El uso de esta metodología en la formación profesional tiene muchos resultados positivos. Por ejemplo, en el 2013, los investigadores Palomer, Humeres, Sánchez, González 
y Contreras concluyeron que el uso de la metodología y las reflexiones de aprendizajeservicio pueden fomentar el desarrollo de valores en los estudiantes (p. 95). Mientras que Seifer y Connors indicaron que el AS "es la metodología perfecta para ser utilizada en la educación superior, pues toma en cuenta las necesidades de los adultos y utiliza métodos y recursos apropiados para facilitar el aprendizaje y el descubrimiento de significado" (2007, p. 6), creando las condiciones perfectas para fomentar el aprendizaje significativo. En el 2002, Kezar concluyó que existe una conexión positiva entre el aprendizaje de servicios y los resultados relacionados con la conciencia cultural, la tolerancia a la diversidad, las actitudes altruistas y el desarrollo moral. Por lo tanto, podríamos inferir que esta metodología podría reducir el etnocentrismo y ayudar a cambiar las opiniones estereotipadas que tienen los jóvenes al trabajar en el desarrollo de la aceptación de la diversidad social y cultural. Estudios e investigaciones más recientes indican que la metodología de aprendizajeservicio fomenta la adquisición de conocimientos conceptuales, y desarrolla habilidades, actitudes y valores sociales en estudiantes de educación superior. Al concluir su estudio, Cooper, Cripps y Reisman afirman que las experiencias de AS afectan las percepciones de los estudiantes como promotores de cambio, creando así profesionales con compromiso cívico y una mirada hacia el servicio comunitario (2013).

En el caso de la profesión docente, esta metodología pareciera ayudar a que los estudiantes estén más conscientes de que su carrera es una profesión al servicio de otros, y que tengan en cuenta las necesidades académicas, personales y sociales, las preocupaciones y los deseos de la comunidad a la que sirven, al tiempo que ofrece la oportunidad de reflexionar sobre la práctica profesional (Santos, Sotelino \& Meledo, 2015, capítulo 1). Opazo, Aramburuzabala y Cerrillo en un estudio sobre AS acerca de la formación del profesorado concluyeron que "había "evidencia de que la adquisición y comprensión conceptual de la materia ocurre de una manera más natural"” $(2016$, p. 81) al utilizar esta metodología, al tiempo que se fortalecen los lazos entre las comunidades y las universidades. Un año más tarde, Mortari, Silva y Girelli presentaron en una conferencia su experiencia con el uso de esta metodología en cursos ofrecidos en la Universidad de Verona en Italia, e indicaron que los profesores o estudiantes-profesores fueron capaces de obtener suficiente conocimiento que les permitía encontrar soluciones a los problemas que enfrentarían en su práctica diaria mientras desarrollaban o mejoraban las habilidades de investigación, ya que tenían la necesidad de encontrar respuestas a las necesidades expresadas por la comunidad. Una investigación llevada a cabo por Adnan y Sinan en el 2015 muestra que el aprendizajeservicio "es un enfoque eficaz para ser utilizado en la educación de los maestros. Existe suficiente evidencia que sugiere que el AS debe formar parte del proceso de instrucción como un enfoque de enseñanza y aprendizaje utilizado activamente" (p. 615). Swick cree que esta metodología ayuda a resolver uno de los desafíos más difíciles de un profesor "que implica entender y actuar sobre el poder que el docente tiene para influir en la vida de los demás" (2001, p. 261). Dado que el AS es la fusión del compromiso institucional del servicio comunitario de los estudiantes, los contenidos académicos de una actividad curricular y la orientación del profesor, parece haber un consenso general de que el AS fomenta la participación e implicación de los maestros y estudiantes en su propia comunidad y fuera del aula mediante el análisis de las necesidades reales, siendo responsable de la toma de decisiones y del diseño de propuestas para tratar de mejorar o transformar estas necesidades. En otras palabras, existe la idea de que esta metodología y sus beneficios son parte del impacto social que el aprendizaje-servicio tiene en sus participantes. El 
impacto social se observa en la influencia y la sostenibilidad en la calidad de vida de las comunidades, mientras que el impacto pedagógico se ve en la mejora del aprendizaje académico a través de la internalización del aprendizaje disciplinario, y se evalúa poniendo en práctica dichos conocimientos. En resumen, la metodología aprendizaje-servicio no sólo fomenta una vida social, política y cívica en los estudiantes de pedagogía, sino que también ayuda a desarrollar las habilidades profesionales necesarias para el siglo XXI, al tiempo que crea un profesional autónomo, reflexivo y crítico que pueda mejorar la imagen que la comunidad tiene de la profesión docente.

Aunque los resultados de otras investigaciones identifican esta modalidad como una metodología que ayuda a las personas a sensibilizarse en el aspecto cultural y social, y a proporcionar una valiosa orientación para la profesionalización de los estudiantes en su fase inicial de formación al tiempo que ofrece prácticas supervisadas, debemos preguntarnos si este impacto positivo incluye a todos los programas de formación del profesorado. Creemos que el uso de esta metodología permitirá a los estudiantes de formación de profesores de EFL desarrollar un sentido social, un respeto por la comunidad y un espíritu de solidaridad, al tiempo que mejora el ejercicio de la profesión. En otras palabras, esta investigación busca identificar si la metodología de aprendizaje-servicio fomenta el desarrollo e internalización de los valores sociales y la profesionalización en los estudiantes mediante la identificación del tipo de impacto que la metodología de aprendizaje-servicio tiene en los estudiantes de formación de maestros de EFL y los miembros de la comunidad en la Región Metropolitana, Chile. Esto se logrará elaborando y poniendo en práctica programas de inglés que cumplan con las necesidades de cada comunidad; identificando cambios en la percepción sociocultural, y el desarrollo profesional y solidario de los estudiantes de formación de EFL mientras guían, asisten y monitorean el trabajo de los estudiantes.

\section{METODOLOGÍA}

Esta investigación es un estudio cualitativo en el que los artículos de revistas se interpretaron por el investigador para dar sentido y encontrar respuestas comunes. A continuación, se describen los participantes, los medios utilizados para medir los objetivos y el procedimiento seguido por los participantes.

\subsection{PARTICIPANTES}

Un total de 290 personas participaron en el estudio. Los participantes, que han sido clasificados como de alto riesgo social o desfavorecidos debido al bajo ingreso y/o condición de inmigrante, se dividieron en dos categorías: el profesor de EFL que capacitaba a estudiantes de pregrado y que representaba una muestra probabilística conveniente, y los miembros de la comunidad y que era una muestra auto-seleccionada no probabilística.

La muestra se compuso de un total de 90 estudiantes de la formación de profesores de EFL divididos en 30 estudiantes de primer año, 30 estudiantes de segundo año y 30 estudiantes de tercer año que se inscribieron en los cursos optativos "Diseño de currículo" y "Diseño de materiales" en el 2016, y 60 estudiantes del primer año inscritos en los cursos "Metodología para enseñar inglés a jóvenes" y "Metodología para enseñar inglés a adolescentes" durante el 2017, 2018 y 2019. Las edades de los participantes oscilaban entre 
los 20 y los 38 años y como parte de los requisitos previos al programa tenían que haber tomado al menos 2 cursos de gramática y 3 cursos de inglés. El muestreo de los miembros de la comunidad estaba compuesto por un total de 200 personas con edades que oscilan entre los 6 y los 35 años, divididos en 20 adultos que trabajaban y 180 estudiantes de primaria y secundaria. En el 2016, hubo 30 participantes de los cuales 10 trabajaban en cafeterías, 10 estudiantes asistían a una escuela de capacitación técnica, 6 participantes trabajaban en un Centro Cultural y 20 estudiantes asistían a una escuela primaria perteneciente al programa "Fortalecimiento de la Educación Pública". Entre el 2017 y el 2019 sólo las escuelas u organizaciones con acuerdos de colaboración podían participar en actividades relacionadas con la práctica debido a los cambios en el Programa Comunitario de la Universidad y la Agencia Nacional de Acreditación, por lo que no fue posible seguir trabajando con los que trabajaban en las cafeterías y centros culturales. Los 150 participantes restantes se matricularon en tres escuelas primarias, una perteneciente a la Congregación Salesiana y dos escuelas secundarias, una de ellas era una Escuela Técnica.

\subsection{HERRAMIENTAS DE EVALUACIÓN}

Se crearon cinco herramientas de evaluación para medir los objetivos, y se utilizaron grupos de debate con todos los participantes. Las tres primeras herramientas fueron creadas por los estudiantes como parte de los requisitos de cada uno de sus cursos, y correspondían a un análisis de necesidades, una encuesta de estilos de aprendizaje del alumno y una prueba de nivelación. El cuarto instrumento, el registro de reflexión, formaba parte del portafolio de evaluación que todos los estudiantes de pregrado tenían que entregar como parte de la evaluación final del curso. El último instrumento, la encuesta de satisfacción, se utilizó para identificar la percepción que la comunidad tenía del aprendizaje-servicio. Los instrumentos fueron validados por los profesores de los cursos y/o escuelas debido a que la creación de la mayoría de los instrumentos formaba parte del programa de los cursos. Los grupos de debates se llevaron a cabo al final de cada curso.

Como se indicó anteriormente, se llevaron a cabo tres tipos de diagnósticos para determinar las necesidades de las comunidades que participaron. Estas herramientas fueron parte del proceso de diagnóstico para determinar las necesidades de los miembros de la comunidad y fueron previamente revisadas por el maestro. La primera herramienta fue una encuesta para determinar las necesidades de las comunidades o "Análisis de las Necesidades". Estas encuestas fueron elaboradas en español y aplicadas por los estudiantes del curso de Diseño curricular. Las encuestas, que constaban de preguntas cerradas y respuestas de opción múltiple intentaron identificar si la comunidad quería aprender inglés debido a razones laborales, escolares o de entretenimiento; lo complicado que les resultaba el idioma; qué material les gustaría usar en las clases y cuánto tiempo podrían dedicar a estas clases. La segunda herramienta determinó el nivel de inglés de las comunidades mediante una prueba oral de nivelación de inglés, elaborada por los estudiantes y revisada por el profesor a cargo de la actividad curricular, utilizando como referencia el Marco Común Europeo (MCER) y los exámenes internacionales de inglés. La tercera herramienta identificó el tipo de estudiantes que asistirían a cada clase y a cada comunidad, utilizando como referencia la teoría de Inteligencias Múltiples de Howard Gardner. Para este diagnóstico, se utilizaron encuestas creadas por Chislett y Chapman (2005-6) y las "Publicaciones ITc". Los datos obtenidos fueron analizados por los estudiantes de formación de profesores de EFL y los 
resultados se utilizaron para diseñar un curso de inglés basado en las necesidades de cada grupo comunitario.

Los diarios de reflexiones tenían un formato de registro con preguntas que guiaron al alumno hacia áreas específicas, como a) acciones positivas de las clases, b) acciones negativas de las clases, c) situaciones o factores que se pueden cambiar inmediatamente y d) situaciones y factores que no se pueden modificar. El objetivo de este registro era alentar a los estudiantes a realizar un seguimiento de su crecimiento personal y profesional mediante el registro de actividades, acciones y comportamientos.

Para medir las percepciones o experiencias negativas y/o positivas de los programas y clases creadas por los estudiantes de pregrado para los miembros de la comunidad, se creó y validó una encuesta por el programa comunitario de la Universidad; ésta tenía 12 afirmaciones y una escala de Likert con números del 1 al 5, donde 1 era muy insatisfecho y 5 estaba muy satisfecho.

Los criterios evaluados en la encuesta fueron:

1. La información previamente ofrecida por la universidad fue clara y precisa.

2. La organización del contenido del curso era coherente con las necesidades establecidas.

3. Se cumplieron las expectativas del curso.

4. El programa del curso proporcionó conocimientos y competencias comunicativas.

5. La actividad o actividades del curso fueron claras y divertidas.

6. La actividad o actividades del curso eran apropiadas para los temas abordados en cada sesión.

7. El profesor dominó el contenido de las clases.

8. El profesor utilizó un lenguaje adecuado y claro a través del desarrollo de las clases.

9. El profesor estableció un ambiente de aprendizaje adecuado.

10. En cuanto a la programación, las clases comenzaron y terminaron a tiempo.

11. El lugar donde se llevó a cabo el curso era cómodo.

12. El curso impartido por la universidad fue interesante y relevante para mi trabajo.

\subsection{PROCEDIMIENTO}

El procedimiento tuvo tres etapas que se repitieron en cada curso y a lo largo de los cuatro años. La primera etapa para los estudiantes de formación de profesores de pregrado de EFL fue crear un "análisis de necesidades", en donde los estudiantes comprobaron y discutieron la validez y eficacia de al menos cinco "análisis de necesidades" antes de elaborar sus propios instrumentos. Las encuestas fueron revisadas por el profesor antes de ser aplicadas a los miembros de la comunidad. Una vez finalizada esta etapa, los alumnos analizaron la información recopilada en las encuestas y presentaron los resultados a sus compañeros. La siguiente etapa fue aplicar una prueba de nivelación en inglés para determinar el nivel de inglés que tenían los miembros de la comunidad, y una vez analizados los resultados, éstos se incluyeron en la elaboración de los programas. La tercera etapa fue la creación de los objetivos del programa y los objetivos que tendría cada unidad, teniendo en cuenta las necesidades y el nivel de inglés de la comunidad. Una vez completada esta etapa, 
los estudiantes presentaron sus programas a las comunidades y comenzaron sus clases. Algunas de estas clases fueron observadas por profesores universitarios para brindar la retroalimentación adecuada y trabajar en las debilidades que se observaron en los estudiantes, mientras que otras fueron observadas por maestros que habían sido entrenados en esta metodología. Finalmente, también se pidió a los alumnos que completaran los registros de reflexión con al menos tres entradas, siendo la primera la que se llevó a cabo durante el desarrollo de los instrumentos, la segunda después de 3 o 4 sesiones con los miembros de la comunidad, y la otra al final del curso. Una vez finalizado el curso, se les pidió a los miembros que completaran la encuesta de satisfacción. Es importante destacar que, a principios de 2017, los miembros de la comunidad eran sólo estudiantes de escuelas debido a la baja participación de la comunidad adulta y los cambios en el Programa Comunitario de la universidad.

\section{RESULTADOS}

Se recolectaron un total de 240 registros de reflexión de los estudiantes de formación de profesores de pregrado de EFL que fueron escritos en el lapso de cuatro años u ocho semestres como parte de su calificación final para cada curso. Los datos se examinaron en búsqueda de respuestas repetitivas y que estaban presentes en más del $50 \%$ de las entradas, en áreas relacionadas con 1) factores que podrían cambiarse, 2) factores que no se pueden cambiar, 3) acciones positivas en la clase/metodología y 4) factores negativos en la clase/metodología. Todas las entradas fueron leídas por el profesor de cada clase y categorizadas en una de las cuatro categorías.

En las entradas que se clasificaron como acciones positivas y que surgieron del uso de esta metodología, una que se encontró en todos los registros fue la recepción positiva que los estudiantes tuvieron al poder desarrollar un instrumento que les ayudara a identificar las necesidades reales y estilos de aprendizaje o formas de procesar la información de la comunidad, y consideraron que esta acción era fundamental para convertirse en profesores eficaces porque les dio la oportunidad de elaborar programas con contenido y actividades que fueran útiles y relevantes para sus estudiantes. Esto era algo nuevo para los estudiantes de pregrado ya que en su experiencia con los cursos de idiomas nadie les había preguntado cuáles eran sus necesidades. Los estudiantes también indicaron que estaban sorprendidos con el alto nivel de participación y compromiso mostrado por las diversas comunidades, e indicaron que incluso si los miembros de la comunidad llegaban tarde o si el horario de clases era después de la jornada escolar o laboral, de igual forma los miembros de la comunidad siempre estaban listos para dar lo mejor de sí mismos. Este último punto, el esfuerzo realizado por las comunidades para completar las tareas, también apareció en más de la mitad de las respuestas. Las reflexiones también mencionan comportamientos profesionales que ser mejorados antes de graduarse y que a primera vista pueden parecer como comentarios negativos, pero tomar conciencia de ellos hace que los profesores sean más eficaces en su labor de enseñanza. Algunas de las entradas más comunes mencionaban mejorar el vocabulario porque no siempre sabían cuál era el vocabulario apropiado. También se dieron cuenta de que la planificación y el tiempo es una de las partes más importantes de la enseñanza, y pudieron ver que el tiempo asignado para las actividades en algunos casos era muy extenso y en otros muy 
corto, quedando entonces poco tiempo para cumplir con el resto de las actividades que estaban planificadas.

En cuanto las respuestas negativas encontradas, la mayoría de los estudiantes de pregrado escribieron que no se sentían cómodos explicando el contenido de la gramática. Tal vez este punto tenga que ver con la eficacia de los enfoques utilizados por sus profesores universitarios en la enseñanza de la gramática. Los estudiantes explicaron que, al explicar los conceptos de gramática, tenían que hacerlo en español y muchas veces los participantes olvidaron las explicaciones o simplemente no entendían lo que estaban tratando de decir. Esto fue bastante frustrante, y muchos estudiantes escribieron largas explicaciones al respecto. Otro aspecto negativo en común era que todavía percibían que sus actividades no eran lo suficientemente lúdicas, y que estaban centradas en el profesor y en la gramática. Esto se percibió como algo muy negativo que se asoció a clases aburridas y en algunos casos a las ausencias de los miembros de la comunidad a las clases. Esta respuesta abundó en los estudiantes universitarios que trabajaban con adultos, y atribuyeron este comportamiento no solo a las actividades aburridas realizadas en clase, sino también en algunos casos a las demandas laborales. Los participantes de la licenciatura también mencionaron que tenían que enseñar en lugares inadecuados como cocinas o cafeterías cuando trabajaban con adultos, y referían que había múltiples interrupciones por parte de los gerentes pidiéndoles a los trabajadores que cubriesen turnos. Estos factores tuvieron un impacto negativo en las sesiones, el proceso de aprendizaje y, por tanto, en la metodología. En el caso de los estudiantes de pregrado que trabajaron con escuelas, ellos destacaron que los directores de las escuelas cambiaron el número de estudiantes y el rango de edad permitido en cada taller, lo que complicó el proceso.

Los resultados de los grupos de discusión con los estudiantes de pregrado revelaron que al principio no estaban contentos con esta nueva metodología, ya que, al leer el programa del curso, parecía duplicar el trabajo. También estaban preocupados por la idea de que iban a ser responsables de diseñar algo para los demás y no sólo de implementar algo ya creado por la universidad. Este fue un sentimiento que era más evidente con los estudiantes que trabajaban con estudiantes adultos o jóvenes. Sin embargo, indicaron que, con el paso del tiempo, se dieron cuenta de que a pesar de que esto era mucho trabajo y a veces temían fracasar, el ver a sus estudiantes hablar o usar inglés en el trabajo hacía que la ardua labor valiera la pena. Al trabajar con adultos, los estudiantes indicaron que se sienten orgullosos y felices de poder ayudar a cambiar la vida de alguien. Los cursos que trabajaban en las escuelas se sorprendieron al ver el apoyo que los jóvenes estudiantes recibían de sus familias, algo que pocos de ellos tenían cuando eran niños. Un tema presente en estas conversaciones, pero no en los diarios, era que les hubiera gustado tener más observaciones de los maestros.

Un factor que no se encontró en los registros, pero fue observado por el profesor a cargo de los cursos universitarios y los profesores que ayudaron en las escuelas, fue que la idea de asistir a las clases de los estudiantes de pregrado y observar las clases sin tener que calificar el rendimiento permitió una mejor y más efectiva retroalimentación, abordando temas en relación al enfoque de enseñanza seleccionado por los estudiantes de pregrado. 
Tabla 1. Gráfico de resultados del análisis de las reflexiones

\begin{tabular}{|l|l|}
\hline Criterios & $\begin{array}{l}\text { Respuestas más frecuentes (que se encuentran en más del } \\
60 \% \text { de los registros) }\end{array}$ \\
\hline a) Acciones positivas de la clase & $\begin{array}{l}\text { Elaborar un instrumento que nos ayude a identificar las } \\
\text { necesidades reales de los miembros. } \\
\text { Los miembros esperaron las clases con entusiasmo, } \\
\text { completaron las actividades y participaron activamente. } \\
\text { Hicieron un gran esfuerzo para lograr lo que les pedimos } \\
\text { que hicieran. }\end{array}$ \\
\hline b) Acciones negativas de la clase & $\begin{array}{l}\text { A veces las actividades eran aburridas para los miembros. } \\
\text { No siempre entendían las explicaciones gramaticales. }\end{array}$ \\
\hline $\begin{array}{l}\text { c) Situaciones o factores que se } \\
\text { pueden cambiar de manera inmediata. }\end{array}$ & $\begin{array}{l}\text { Mejorar mi vocabulario porque no siempre sabía lo que } \\
\text { estaban pidiendo. } \\
\text { suficiente tiempo para hacer todo lo que estaba planeado. } \\
\text { Las clases eran demasiado cortas. }\end{array}$ \\
\hline $\begin{array}{l}\text { d) Situaciones o factores que no se } \\
\text { pueden cambiar }\end{array}$ & $\begin{array}{l}\text { El lugar no siempre fue apropiado. } \\
\text { La baja asistencia de los miembros a las clases. }\end{array}$ \\
\hline
\end{tabular}

Fuente: Elaboración propia.

Por último, el análisis de los resultados de la encuesta de satisfacción de los miembros de la comunidad mostró que el 50\% estaba de acuerdo en que la organización de contenidos era coherente con las necesidades previamente establecidas, el 66\% de los miembros indicaron que se cumplían las expectativas que tenían del curso, mientras que el 17\% indicaba que no se cumplían. El $72 \%$ de los miembros indicaron que los programas contribuyeron a sus conocimientos y competencias comunicativas en inglés y que las actividades del curso eran claras y divertidas. El 66\% estuvo de acuerdo en que las actividades del curso eran apropiadas para los temas abordados en cada sesión, mientras que el 11\% de los miembros de la comunidad indicaron que los profesores no dominaban el contenido de la clase. Es importante mencionar que, aunque se programaron reuniones para discutir en profundidad estas respuestas, los miembros de la comunidad no fueron y argumentaron que no tenían tiempo para asistir.

Lamentablemente, no fue posible llevar a cabo la reunión de discusión con los grupos comunitarios debido a la falta de autorización de las escuelas. 
Estudios Pedagógicos XLVI N 3: 93-109, 2020

IMPACTO DE LA METODOLOGÍA DE APRENDIZAJE-SERVICIO DEL PROGRAMA DE GRADO DE CAPACITACIÓN DE PROFESORES DE INGLÉS EXTRANJERO PARA ESTUDIANTES CHILENOS DESFAVORECIDOS

Figura 1. Resultados de la encuesta de los miembros de la comunidad ${ }^{1}$

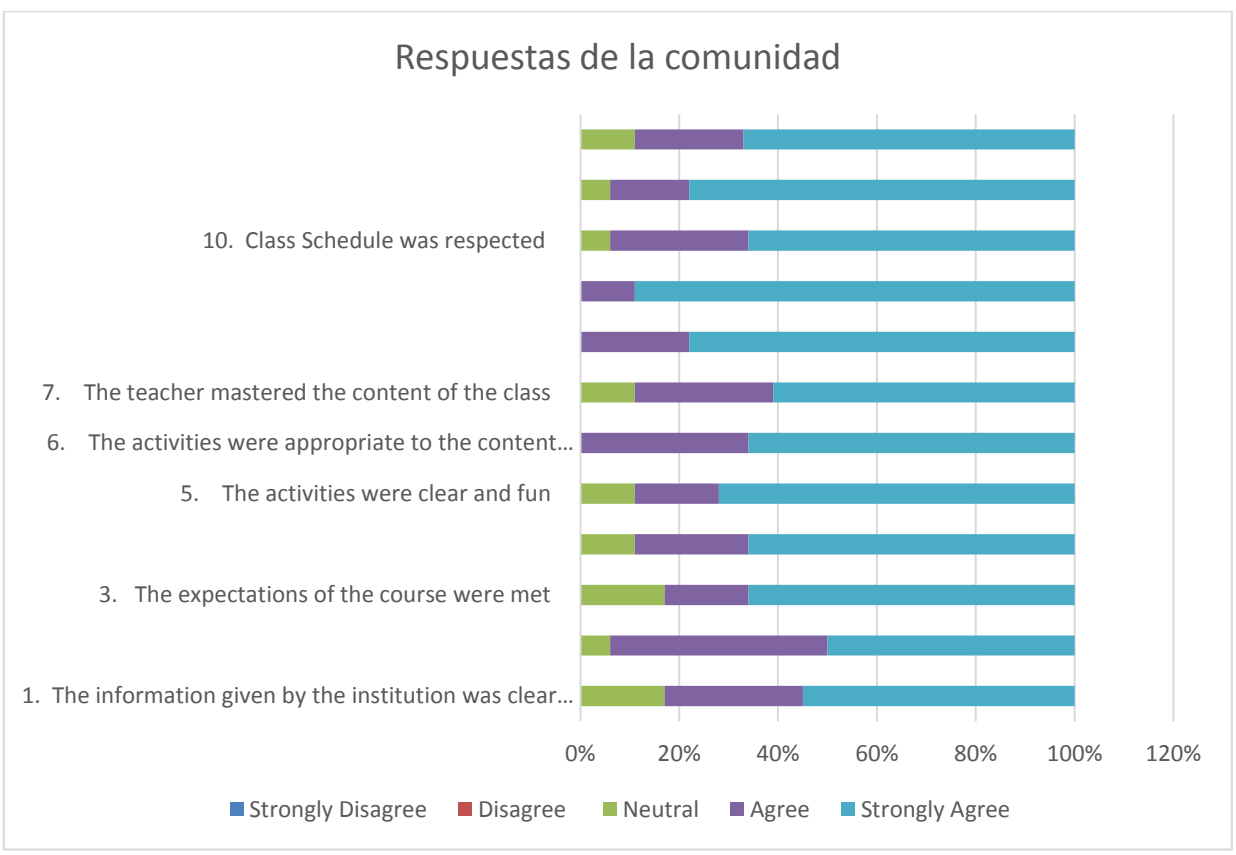

Fuente: Elaboración propia.

\section{DISCUSIÓN}

Los resultados de los registros de reflexión y las encuestas de satisfacción indican que la metodología de aprendizaje-servicio en el programa de formación de profesores de pedagogía inglesa ha sido satisfactoria y ha cumplido con las expectativas académicas y sociales establecidas en los programas al inicio de cada período. Un análisis más detallado en el análisis de los resultados indica que los estudiantes inscritos en estos cursos estuvieron más conscientes de lo que significa ser un profesor eficiente y eficaz al considerar las necesidades de las personas para lograr objetivos personales, planificar clases y actividades basadas en términos de contenido como gramática, vocabulario, etc. En otras palabras, hay evidencia

\footnotetext{
12. El curso fue interesante y me ayudó en mi trabajo

11. El lugar donde se llevó a cabo el curso era cómodo

10. Se respetó el horario de la clase

9. El profesor creo un ambiente cómodo y seguro

8. El profesor utilizó un lenguaje apropiado

7. El profesor manejó el contenido de la clase

6. Las actividades se diseñaron de forma apropiada de acuerdo al contenido

5. Las actividades eran claras y divertidas

4. El programa promovió el conocimiento y las habilidades comunicativas

3. Se cumplieron las expectativas del curso

2. El contenido se organizó de forma coherente

1. La información brindada por la institución fue clara.
} 
que apoya los hallazgos de Opazo, Aramburuzabala y Cerrillo al indicar que el uso de esta metodología permite "la adquisición y comprensión conceptual de la materia de una manera más natural” (2016, p. 81) y no sólo en un estilo de clase tipo conferencia que tiende a ser un poco artificial. Los resultados también apoyan los hallazgos de Ling con estudiantes universitarios desfavorecidos de primera generación, al referirse que los estudiantes sentían que este tipo de metodología les ayuda a desarrollar habilidades y comprensión, desarrollar resiliencia, encontrar significado personal y desarrollar la conciencia crítica (2010, hallazgos).

En resumen, la innovación educativa que ofrece este modelo aporta los siguientes beneficios:

1. Permite a los estudiantes tener más práctica en situaciones de la vida real.

2. Ofrece a los estudiantes la posibilidad de desarrollar competencias profesionales.

3. Crea un entorno de aprendizaje colaborativo.

4. Crea un vínculo con las comunidades vecinas.

5. Relaciona al estudiante en el proceso de identificación de un problema, elaboración de una solución, y aplicación de la misma.

6. Crea una cultura de solidaridad.

Esta investigación hace énfasis en la importancia del trabajo colaborativo entre los estudiantes, las universidades y los miembros de la comunidad, donde la relación entre todas las partes ayuda a crear profesionales mejor preparados, con herramientas adecuadas para la realidad de cada país o región. Como afirman Folgueiras, Luna y Puig, "existe una alta y buena percepción sobre los conocimientos conceptuales, personales y cívicos adquiridos por los estudiantes" (2013, p. 12) durante esta metodología.

Los resultados también muestran que es importante resaltar que los profesores universitarios deben salir del aula universitaria, abandonar sus clases y participar en actividades donde asuman el papel de guías o modelos en situaciones de la vida real, mostrando a los estudiantes sus habilidades, intereses, pasión y motivación al trabajar en un contexto de la vida real (Garcia-Perez \& Mendia, 2015, p. 44).

Como profesores de idiomas, el poder ser parte y estar dentro de la comunidad en un papel activo y no sólo como observadores, nos ayuda a identificar las estrategias, metodologías y materiales más adecuados para aprender un segundo idioma, así como reconocer prácticas efectivas en la gestión del aula. Otro factor importante que aparece en este estudio es la eficacia de la retroalimentación inmediata. Es importante resaltar que la retroalimentación es a veces más relevante que la adquisición del conocimiento, porque en este proceso el estudiante y el profesor tienen la oportunidad de aprender unos de otros. Como dijo Swick, es a través de "el aprendizaje mutuo que ocurre en los proyectos de tutoría, cuando el profesor y el alumno obtienen nuevos conocimientos y crecen juntos" (2001, p. 261). Como profesora responsable de responder a las necesidades cambiantes de nuestra sociedad, es fundamental proporcionar a tiempo una retroalimentación a los futuros maestros, resolver los problemas o responder las dudas que aparecen durante las clases, con el fin de minimizar las prácticas que tienen poca efectividad o son inapropiadas para el contexto.

Estas reflexiones, vistas no como piezas individuales de escritura sino como parte de un portafolio o un registro de crecimiento personal y profesional, evidencian que los estudiantes de formación de profesores de pregrado de EFL han entendido la importancia de saber qué tipo de estudiantes tienen en un aula y qué esperar de las actividades o clases. 
IMPACTO DE LA METODOLOGÍA DE APRENDIZAJE-SERVICIO DEL PROGRAMA DE GRADO DE CAPACITACIÓN DE PROFESORES DE INGLÉS EXTRANJERO PARA ESTUDIANTES CHILENOS DESFAVORECIDOS

Los estudiantes de pregrado de EFL también fueron capaces de apreciar la importancia de tener objetivos claros, preparar clases, material y actividades que fueran lúdicas, contextualizadas a la realidad de cada comunidad antes de dictar una clase. Como ha concluido el alcalde Paredes, este tipo de metodología permite a las universidades:

Proporcionar escenarios que fomenten el desarrollo de las habilidades personales, estudiantiles y sociales necesarias para diagnosticar e intervenir en el mundo real, y diseñar actividades de reflexión enfocadas en mejorar el propio análisis y aumentar el compromiso personal y social de los estudiantes (2018, p. 19).

De los resultados del grupo de discusiones podemos inferir que los estudiantes comenzaron a interiorizar el gran poder que tienen los profesores para influir en la vida de los demás y en algunos momentos de la vida de las personas para cambiarlos para mejor. Los resultados también nos permiten ver cómo los estudiantes están interiorizando lo que significa ser un profesor y poder tomar muy en serio la responsabilidad que tenemos.

Como en cualquier otro estudio, este tiene limitaciones que tienen que ver con factores y situaciones que ocurrieron y que podrían haber afectado los resultados del estudio. Algunos de ellos son:

1. Cambios en las regulaciones y el perfil de la muestra: Debido a que hubo un cambio en el programa comunitario de la Universidad y en los requisitos de la Agencia Nacional de Acreditación que regula o guía en la descripción de los "miembros comunitarios", muchos de los participantes adultos no podían seguir siendo parte del programa ya que no había ningún documento legal vinculante que garantizara la participación.

2. Proceso y análisis de recopilación de datos: Otra limitante que se puede observar es que, debido a que los datos forman parte de un curso que busca desarrollar habilidades relacionadas con la disciplina de "enseñar inglés" y que necesita responder sobre el proceso de enseñanza, el análisis de la información es difícil y es dejado a la interpretación del profesor. En el futuro sería más conveniente agregar entrevistas en profundidad y grupos focales como parte de la recopilación de los datos.

3. Disturbios sociales: Durante el 2019 debido al estallido social y las constantes protestas que afectaron al país, muchos programas tuvieron que ser suspendidos por lo que se recogieron datos insuficientes.

4. Tiempo y falta de participación en entrevistas por parte de la comunidad: Los participantes estaban reacios a participar en las entrevistas realizadas al final de las clases, indicando que no tenían tiempo para participar o en el caso de las escuelas que no tenían autorización de los padres. Esto fue una sorpresa ya que cuando aceptaron participar en el programa se les mencionó esta actividad.

\section{CONCLUSIÓN}

La metodología propuesta con este modelo de intervención nos ha permitido impulsar nuevas formas de trabajar en los programas iniciales de formación del profesorado, estableciendo una relación directa y multidireccional entre la teoría de la práctica docente, 
la aplicación de conceptos teóricos y las necesidades reales tanto de las comunidades vecinas como de los profesores en formación. Como señaló Kaplún, "estas múltiples formas de interactuar con la sociedad son oportunidades de aprendizaje significativo, pues enfrentan a los profesores y estudiantes a problemas concretos y complejos que deben ser resueltos" (como se cita en Santander, 2019, p. 16). Al inicio del estudio, los estudiantes de pregrado de formación de EFL tenían una idea diferente de cuál era el verdadero trabajo del profesor, sus diferentes roles y posibilidades de ser agentes de cambio que podrían generar una transformación social. Pero después de completar el curso pudieron descubrir que escuchar a las comunidades, entender lo que necesitan y cómo procesan la información les permite crear programas adecuados para la comunidad donde fueron capaces de innovar en las formas de enseñanza y materiales usados. Para algunos estudiantes, esta metodología fue el inicio de una nueva vida en la que se ven a sí mismos como agentes de cambio social que podrían ayudar a transformar la vida de los demás. Para otros estudiantes este es sólo otro curso con una metodología diferente que necesitaban aprobar para graduarse.

A pesar de que los resultados han demostrado que esta metodología tiene un impacto positivo en la formación de profesores de pregrado de EFL, sería interesante identificar:

1. Si este efecto positivo se mantiene en el tiempo en estos futuros profesores, mediante la implementación de esta metodología en algunas de sus clases o trabajo.

2. Los efectos de esta metodología en los miembros comunitarios y sus familias.

3. La inclusión de las TICs en esta metodología.

Como profesora que ha utilizado esta metodología durante los últimos 5 años, es cierto que su implementación requiere mucha planificación, reunión con los estudiantes, visitas a las comunidades y un incremento en el número de tareas que tienen que ser abordadas y/o corregidas. Sin embargo, el hecho de ver cómo mis alumnos se han vuelto más conscientes socialmente, respetuosos de las diferentes realidades de los diversos grupos de las comunidades, al observar que han mejorado sus prácticas profesionales al tiempo que mejoran las oportunidades de trabajo de los miembros comunitarios, en el caso de trabajar con adultos, ha sido inspirador y vigorizante. Mi experiencia personal con esta metodología apoya los hallazgos de Boyle-Baise (Santander 2019, p. 49) que demostraron que esta metodología crea un mayor compromiso con el proceso de enseñanza. El uso de la metodología aprendizaje-servicio ha sido una gran oportunidad para generar espacios de trabajo con las comunidades que nos rodean, permitiéndonos escuchar los unos a otros y entender que el futuro de todas las sociedades es algo que se construye trabajando juntos y no como instituciones aisladas.

\section{REFERENCIAS BIBLIOGRÁFICAS}

Adnan, K. \& Sinan, K (2015). The Effectiveness of Community Service Learning in Teacher Training. Journal of Theory and Practice in Education, 11(2), 610-629.

Alliance for Service-Learning in Education Reform (1993). Standards of Quality for School-based Service-Learning. Chester, VT: Alliance for Service-Learning in Education Reform. 
Estudios Pedagógicos XLVI N 3: 93-109, 2020

IMPACTO DE LA METODOLOGÍA DE APRENDIZAJE-SERVICIO DEL PROGRAMA DE GRADO DE CAPACITACIÓN DE PROFESORES DE INGLÉS EXTRANJERO PARA ESTUDIANTES CHILENOS DESFAVORECIDOS

Berríos, V., Contreras, M., Herrada, M., Robles, M. y X. Rubio, (2012). Resultados de Aprendizaje Servicio en la UC: informe final de investigación. Retrieved from https://scielo.conicyt.cl/scielo. php?script=sci_arttext\&pid=S0718-45652013000200007

Bringle, R. G. \& Hatcher, J. A. (1995). Institutionalization of service-learning in higher education. Journal of Higher Education, 71(3), 273-290.

Contreras, M., (2010) Modelo de sistematización: una herramienta para sistematizar cursos que implementan Aprendizaje Servicio en la UC. Retrieved from https://scielo.conicyt.cl/scielo. php?script=sci_arttext\&pid=S0718-45652013000200007

Cooper, S., Cripps, J. y Reisman, J. (2013). Service-learning in deaf studies: impact on the development of altruistic behaviors and social justice concern. American Annals of the Deaf, 157(5), January: 413-27.

Chislett, V. \& Chapman A. (2005-6). Multiple Intelligences Test - based on Howard Gardner's MI Model. Retrieved from http://www.businessballs.com/freepdfmaterials/free_multiple_ intelligences_test_manual_version.pdf

Folgueiras, P., Luna, E. \& Puig, G. (2013) Service Learning: Study on the Degree of Satisfaction of University Students. Revista de Educación, 362. Retrieved from http://www.revistaeducacion. educacion.es/doi/362_157.pdf

Furco, A. \& S, Billig, (2002). Service learning: the essence of pedagogy. Connecticut: IAP.

García-Perez, A. \& Mendía, R. (2015). Acompañamiento Educativo: el rol del educador en aprendizaje y servicio solidario. Profesorado. 19(1).

Groh C., Stallwood L. \& J, Daniels, (2011). Service-learning in nursing education: its impact on leadership and social justice. Nurse Education Perspectives, 32(6), 400-405.

ITC Publications, (2014). The Multiple Intelligence Quiz. Retrieved from http://www.collegesuccess 1. com/InstructorManual4thEd/Learning\%20Style/MI_quiz.pdf

Kahne, J., Westheimer, J. \& Rogers, B. (2000). Service Learning and citizenship in higher education. Michigan Journal of Community Service Learning, 42-51.

Kezar, A. (2002). Assessing community service-learning: Are we identifying the right outcomes? Sage Journals, 7(2), 14-20.

Ling, T., (2010). Service Learning and persistence of low-income, fist-generation college students: An exploratory study. Michigan Journal of Community Service Learning, 50-65.

Mitchell, T. (2008). Traditional vs. critical Service-Learning: Engaging the literature to differentiate two models. Michigan Journal of Community Service Learning, 500-65.

Mortari, L., Silva, R. \& Girelli, C. (2017) Community Service Learning Experiences In PreService Teachers' Training. Paper presented in the International Technology, Education and Development Conference. Retrieved from/publication/315353542_COMMUNITY_SERVICE_ LEARNING_EXPERIENCES_IN_PRE-SERVICE_TEACHERS\%27_TRAINING

Newman, C. y Hernández, S. (2011). Minding our business: longitudinal effects of a service-learning experience on alumni. Journal of College Teaching and Learning, 8(8), August, 39-48.

Opazo, H., Arramburuzabala, P. \& Cerrillo, R. (2016). A review of the situation of service-learning in higher education in Spain. Asia-Pacific Journal of Cooperative Education, 17(1), 75-91.

Palomer, L., Humeres, P., Sanchez, A., Gonzalez, S. \& Contreras, A. (2013). Una experiencia de "aprendizaje-servicio": fomentando el desarrollo de valores en estudiantes de odontología chilenos. FEM, 16(2), 91-96.

Paredes, D. (2018). Aprendizaje-Servicio: una practicas educativa innovadora que promueve el desarrollo de competencias del estudiantado universitario. Revista Electrónica Actualidades Investigativas en Educación, 18(3).

Saltmarch, J. (1996). Education for critical citizenship: John Dewey's contribution to the pedagogy of community service learning. Michigan Journal of Community Service Learning. Fall 1996, 13-21.

Santander, G. (2019). Compartiendo una mirada del aprendizaje y servicio. Santiago: Ediciones Universidad Santo Tomás. 
Santos, M., Sotelino, A. \& Moledo, M. (2015). Aprendizaje -servicio y misión cívica de la universidad. Una propuesta de desarrollo. Barcelona: Editorial Octaedro.

Seifer, SD. \& Connors, K. (2007) Faculty Toolkit for Service-Learning in Higher Education. California: National Service-Learning Clearinghouse.

Swick, K. (2001) Service-Learning in teacher education: Building Learning Communities. The Clearing House: A Journal of Educational Strategies, 74(5), 261-264.

Tapia, M. (2006). La solidaridad como pedagogía. Ciudad Nueva: Buenos Aires. . (2016). XIX Seminario Internacional de Aprendizaje y Servicio Solidario. Buenos Aires: CLAYSS.

UNESCO Institute for Lifelong Learning. (2015). The Role of Higher Education in Promoting Lifelong Learning. Germany: UIL.

Vázquez Verdera, V. (2015). El aprendizaje-servicio: una estrategia para la formación de competencias en sostenibilidad. Foro de Educación, 13(19), 193-212.

Wade, R. (2000). Building Bridges: Connecting Classroom and Community through ServiceLearning in Social Studies. ERIC- NCSS Bulletin 97. 
